\title{
Les facultés de lettres de province dans la tourmente des réformes de l'enseignement supérieur (1896-1914)
}

Provincial Arts Faculties in the turmoil of the higher education reforms

(1896-1914)

\section{Evelyne Héry}

\section{OpenEdition \\ Journals}

Édition électronique

URL : http://journals.openedition.org/abpo/2495

DOI : $10.4000 / a b p o .2495$

ISBN : 978-2-7535-2236-7

ISSN : 2108-6443

Éditeur

Presses universitaires de Rennes

\section{Édition imprimée}

Date de publication : 31 décembre 2012

Pagination : 83-98

ISBN : 978-2-7535-2234-3

ISSN : 0399-0826

Référence électronique

Evelyne Héry, «Les facultés de lettres de province dans la tourmente des réformes de l'enseignement supérieur (1896-1914) », Annales de Bretagne et des Pays de l'Ouest [En ligne], 119-4 | 2012, mis en ligne le 31 décembre 2014, consulté le 02 mai 2019. URL : http://journals.openedition.org/abpo/2495 DOI : 10.4000/abpo.2495 


\title{
Les facultés de lettres de province dans la tourmente des réformes de l'enseignement supérieur (1896-1914)
}

\author{
Évelyne HÉRY \\ maître de conférences en histoire contemporaine, \\ université Rennes 2, CERHIO UMR 6258
}

"Les malheureuses facultés de province ", ne faut-il voir qu'un lieu commun dans la reprise en 1904 par Charles Dupuy, ancien ministre de l'Instruction publique et ancien chef de gouvernement, de cette expression que Renan avait employée en 1875 pour désigner les facultés de lettres de province? Ou, moins fréquentées que les facultés "professionnelles ", c'està-dire les facultés de droit et de médecine, et moins assurées que leurs voisines de sciences de pouvoir diversifier les débouchés de leurs formations, les facultés de lettres restent-elles défavorisées au sein des universités que la loi du 16 juillet 1896 a créées ${ }^{1}$ ? Leurs faibles effectifs peuvent le laissent penser puisque, depuis qu'a été fixé le statut d'étudiant inscrit ${ }^{2}$, le nombre détermine le montant des moyens alloués. En tout cas, leurs doyens et leurs personnels affirment être victimes des nouvelles réformes et transmettent leurs doléances à la Société pour l'étude des questions d'enseignement supérieur dont la Revue internationale de l'enseignement (RIE) est l'organe. La revue donne largement la parole aux quinze universités provinciales; elle publie les réactions de leurs enseignants à l'actualité, les discours des rentrées solennelles et les rapports annuels d'activité, et ce d'autant plus que, pour son nouveau comité de rédaction entré en fonction au lendemain du vote de la loi, dirigé par F. Picavet et constitué - comme l'ancien - exclusivement de membres d'établissements supérieurs parisiens, le souci de rester à l'écoute de la communauté universitaire pour ne rien perdre de

1. Voir Annexe 1, la liste des facultés et leurs effectifs. La loi de 1896 érige en universités, toutes dotées des mêmes droits et prérogatives, les corps de facultés qui avaient été créés en 1893 au niveau académique et disposaient de la personnalité civile et d'un budget propre.

2. Le statut d'étudiant inscrit, selon des formalités réglementées par décret, va de pair avec la création dans les facultés de lettres et de sciences de cours "fermés ", par opposition aux cours publics, suivis par des auditoires libres. 
son influence auprès des pouvoirs publics passe par la relance de l'activité des groupes de province ${ }^{3}$. Dans une moindre mesure, la Revue universitaire $(R U)$ se fait aussi l'écho des débats.

À partir du corpus des documents cités ci-dessus, la question qui m'a retenue est contenue dans l'expression même de "malheureuses facultés de province ". En quoi la restructuration de l'Université et les modifications importantes qui, comme on le verra plus loin, affectent la préparation du professorat de l'enseignement secondaire après la réforme de l'ENS (École normale supérieure) en 1903 ont-elles pu réactiver l'image misérabiliste qui était associée aux facultés de lettres, Sorbonne exceptée? Si, organisées autour d'un enseignement de culture générale qui ne débouche guère que sur le professorat de l'enseignement secondaire, elles sont effectivement confrontées à la pénurie d'étudiants et se trouvent exposées à la concurrence de Paris dans leurs efforts pour se développer et œuvrer à l'avancement de la science, comme le stipulait la loi, sont-elles restées en marge de la renaissance de l'enseignement supérieur et, avec lui, de l'enseignement universitaire des lettres?

\section{Des facultés sans étudiants ${ }^{4}$ : la crainte de dépérir}

Le faste des fêtes d'inauguration et des séances de rentrée des nouvelles universités, largement relatées dans la $R I E$, peut masquer, dans un premier temps, les inquiétudes qui tempèrent la satisfaction de voir réalisée la réforme de l'université. Mais F. Lot donne le ton : "On vient de reconnaître officiellement nos universités françaises. À quoi bon cette étiquette si nos Facultés des sciences, et surtout des lettres, continuent d'être pourvues d'un personnel aussi maigre, si quantité de disciplines sont systématiquement ignorées ${ }^{5}$ ? "Certes, F. Lot a toujours été très critique à l'égard de l'enseignement supérieur français mais la crainte que la mise en œuvre de la loi achoppe sur la réalité de l'inégalité des universités de province, et en leur sein, sur la situation critique des facultés de lettres est exprimée par d'autres universitaires. A fortiori, les facultés de lettres des "petites universités ${ }^{6}$ " dont, au sein des milieux universitaires et politiques, la reconnaissance est vivement controversée semblent en mauvaise posture parce qu'elles ont peu de professeurs et peu d'étudiants. C'est le cas de Clermont notamment qui, comme Besançon, n'appartient pas à une université « complète ", et Ehrhard et Hauser posent nettement, dès 1897, dans la Revue

3. PICAVET, François, "Lettre aux recteurs et doyens des Universités ", RIE, 1897, 1, p. 386. Par cette lettre, le nouveau comité de rédaction entré en fonctions en 1897 se propose "de solliciter toutes les actions qui pourront lui venir des groupes de province ".

4. EHRARD, Albert, HAuSER, Henri, "L'avenir et le rôle d'une petite université ", Revue Universitaire, 1897, 1, p. 225-236.

5. Lot, Ferdinand, "Deux universités : Strasbourg et Nancy", RIE, 1896, 2, p. 138 - 141.

6. Audollent, Alphonse, "Encore un mot sur les petites universités ", RU, 1898, 1, p. 213245. L'expression de "petites universités " est mise entre guillemets et en italique dans l'article, mais Audollent note qu'elle est devenue d'usage courant en 1898. 
universitaire la question de leur avenir ${ }^{7}$. D’une façon générale, la statistique du nombre d'étudiants immatriculés dans les facultés des lettres au lendemain de la réforme montre la faiblesse des effectifs pour la majorité des établissements de province ${ }^{8}$. En histoire, une discipline où l'augmentation est pourtant nette, Rennes avec une dizaine d'étudiants en licence se situe dans la moyenne. Mais souvent, le nombre d'étudiants " réels ", c'est-à-dire se présentant aux examens est encore inférieur, certains n'étant inscrits que pour ne faire qu'un an de service militaire.

En 1910, M. Rainaud, professeur à la faculté des lettres de Caen pouvait résumer le problème général des universités de province en ces termes : "Il nous faut des étudiants, il nous faut de l'argent pour développer nos laboratoires, nos collections, nos enseignements, pour perfectionner notre outillage de travail ${ }^{9}$. " En effet, chaque faculté, en vertu de l'article 4 de la loi de 1896, conservant ses ressources propres, constituées par les droits d'études et d'inscription acquittés par les étudiants, pour couvrir les dépenses de laboratoire, de bâtiments et la création de nouveaux enseignements, son activité était, pour partie, suspendue au nombre d'étudiants qu'elle immatriculait. On comprend dès lors que le spectre de la banqueroute ait été brandi et que les facultés des lettres aient cherché à recruter au-delà de leurs viviers " naturels ", à savoir les candidats à l'enseignement et les étudiants libres. Cela a été le cas pour les étudiants étrangers, quasi-absents en province alors qu'à Paris, ils constituaient 6 \% de l'effectif total des immatriculés en 1898. Très tôt, les efforts des universités provinciales ${ }^{10}$ se sont portés dans cette direction, riche de perspectives pour les facultés de lettres qui enseignaient par définition la littérature et la langue françaises. Dans ce domaine, la possibilité offerte aux universités par les décrets d'application de 1897 de décerner des titres spécialement créés par elles, distincts des grades d'État, et, à partir de la loi de finances de 1899, de percevoir les rétributions afférentes en matière de droits d'études et d'examens, a donné lieu à des créations de certificats d'études françaises, de littérature française, et de doctorats d'université.

Pour les facultés de lettres, il s'est aussi agi de conquérir le public féminin à l'heure où la demande de formation des jeunes filles était en plein essor et où elles étaient de plus en plus nombreuses à vouloir acquérir

7. EhraRD, Albert, HAuSER, Henri, $R U, 1897,1$, p. 228. Ce faisant, Ehrhard et Hauser, qui enseignent alors à Clermont, ouvrent un débat. Le recteur Zeller, dans la séance de rentrée de l'Université en 1898, nuance leur analyse. Déclarant, pour rassurer le monde politique local, que l'université de Clermont n'a pas lieu de se croire en danger, il précise : «L'université de Clermont n'est pauvre que par comparaison de ses modestes ressources avec les richesses scientifiques depuis longtemps accumulées dans les grandes villes par ce mouvement centralisateur que tout le monde déplore et que tout le monde pratique ", RIE, 1898, 2, p. 534 .

8. Voir Annexe 1.

9. « Rapport de la faculté des lettres de Caen pour l'année 1908-1909 » Caen, RIE, 1910, 2, p. 162-164.

10. On voit très nettement dans l'annexe 1 que les facultés des provinces frontalières ont particulièrement joué cette carte. 
des diplômes, voire les mêmes grades que leurs camarades masculins. Si elles ne sont encore que 133 jeunes filles à être immatriculées le 15 janvier 1898 dans les quinze facultés des lettres de province, cinq facultés n'en comptant aucune et cinq moins de dix, alors que la Sorbonne à elle seule en compte 129, la croissance des effectifs féminins dans l'enseignement supérieur et notamment dans les études supérieures de lettres entre 1900 et 1914 ouvre des perspectives nouvelles ${ }^{11}$.

Enfin, les facultés de lettres, comme celles de sciences, ont cherché dans l'extension universitaire de nouvelles sources de recrutement. Sur le modèle anglo-saxon dont la RIE tenait ses lecteurs informés, l'extension universitaire était une extension des activités de la faculté due notamment à l'élargissement de son offre d'enseignement à de nouvelles catégories d'étudiants, conformément au pouvoir conféré au conseil d'université par le décret du 21 juillet 1897 d'organiser des cours : par exemple la faculté de Toulouse ouvre les études universitaires aux candidats aux concours de Saint-Cloud et Fontenay ${ }^{12}$; Dijon propose d'assurer des cours destinés aux professeurs des EPS (École primaire supérieure) ou du primaire ${ }^{13}$, et met en place, après la réforme de 1902, une année de culture générale commune aux bacheliers inscrits en lettres et en droit qui permet de drainer un public plus nombreux ${ }^{14}$. Si Dijon mise là sur un enseignement interfacultaire de culture générale, prônant, somme toute, une " mutuellisation " des moyens, une extension universitaire "interne ", l'idée d'une année de propédeutique à la licence fait également son chemin, soutenue par la majorité des facultés ${ }^{15}$, avant même l'institution des licences spécialisées par le décret du 8 juillet 1907 qui légitime encore plus le projet. Il est enfin possible, à l'instar des facultés de sciences, de miser sur le public local en continuant à développer des enseignements et des recherches qui participent à l'éducation populaire ou/et répondent à la curiosité de la société cultivée. C'est la ligne que préconise Hauser en écrivant dans la Revue universitaire que les facultés de provinces doivent " [ s'appliquer] aux recherches qu'elles peuvent poursuivre mieux que toutes leurs rivales et qui ne peuvent être menées à bien que chez elles et par elles ". Ainsi, entre autres exemples, à Clermont l'enseignement de l'histoire de l'Auvergne, à Grenoble celui de l'histoire du Dauphiné et un de géographie alpine, à Rennes, celui de langue et de littérature celtiques ont-ils été créés et ont-ils prospéré.

11. CondetTE, Jean-François, "Les Cervelines " ou les femmes indésirables, l'étudiante dans les années 1880-1914, Carrefours de l'Education, 2003/1, n 15, p. 38-61.

12. "Les universités françaises en 1912-1913 ", RIE, 1914, 2, p. 213.

13. "Séance de rentrée de l'université de Dijon ", RIE, 1904, 2, p. 553-560.

14. Plusieurs universités examinent ce projet au début du siècle; TouTAIN, J., "Création dans les universités françaises d'une année de préparation aux facultés de droit et de lettres ", RIE, 1901, p. 206-211.

15. "Organisation dans les facultés de lettres d'une année d'études préparatoires de l'enseignement supérieur ", Rapport présenté à la faculté des lettres de Lyon par sa commission des réformes, RIE, 1906, 1, p. 131. 
On assiste donc à une diversification de l'offre d'enseignement mais les facultés de lettres de province bénéficient moins que la Sorbonne de la croissance des effectifs de l'enseignement supérieur. Pour l'année universitaire 1912-1913, la Sorbonne immatricule en lettres presque dix fois plus d'étudiants que la plus importante des facultés de province. Par ailleurs, la croissance de leurs effectifs étudiants ne signifie pas toujours une croissance équivalente de leur activité en raison de la surévaluation due à l'immatriculation des étudiants étrangers. Ou bien l'organisation de cours de vacances, comme en propose Rennes, ne dure que quelques semaines. Pourtant, toutes insistent sur les échanges avec les universités étrangères et les recrutements qu'elles ont faits. Grenoble s'enorgueillit ainsi d'être devenue la première des facultés de lettres de province, Caen se félicite en 1912-13 de compter 34 étudiants étrangers, dont 30 britanniques. La réalité est que la plupart, sauf Lyon, Bordeaux et Lille ont une population de boursiers de licence et d'agrégation insuffisante pour soutenir leur activité et obtenir la moindre création de postes ${ }^{16}$. C'est pourquoi la réforme de l'ENS en 1903 a été perçue comme un coup fatal.

\section{Le conflit avec Paris}

Si une relative expectative sur les retombées de la loi régnait aux lendemains de 1896 - hormis pour les " petites " facultés qui s'étaient, on l'a vu, très tôt inquiétées de leur sort -, l'examen au Sénat de la suppression des dispenses du service militaire qui fait craindre une baisse des effectifs, et surtout la réforme de l'ENS par le décret du 10 novembre 1903, la réunissant à l'Université de Paris et modifiant les conditions d'admission, provoquent de la part des facultés de province une riposte immédiate dont Léon Clédat, doyen de la faculté des lettres de Lyon, la plus importante par le nombre d'étudiants immatriculés après Paris, s'institue en quelque sorte le porte-parole. Manifestement, l'indétermination du nombre d'élèves admis à l'École normale supérieure crée une forte impatience car l'article $6 \mathrm{du}$ décret stipulant qu'il "ne peut être inférieur au chiffre moyen des agrégés reçus au concours pendant les cinq dernières années ", il est possible d'en déduire que la réforme augmente en fait le nombre des futurs normaliens, par rapport aux vingt qui avaient été jusqu'alors admis. Or, en vertu de l'article 7, il faut ajouter à ces pensionnaires de l'École normale, les «boursiers externes " mis à sa disposition. Dans ce climat tendu où une grande part de l'argumentation en faveur ou non de la réforme repose sur des supputations, le décret du 10 mai 1904, fixant que, à partir de la rentrée 1905, le recrutement des élèves de l'École et des boursiers de licence s'opèrera par le même concours national, est mal reçu. Il apparaît d'abord comme une atteinte à la souveraineté des universités provinciales qui, avant, organi-

16. "Enquête sur la spécialisation des facultés de lettres et l'agrégation de philosophie ", $R I E, 1909,2$, p. 148-161. Bien que moindre à la fin de la décennie 1900-1910, la pénurie des étudiants en province reste préoccupante d'après les réponses que les doyens adressent au questionnaire sur la spécialisation, 1909, 2, p. 238-249; 363-380; 410-412. 
saient leur propre concours de bourses, et surtout comme une entreprise de ponction des meilleurs étudiants de province.

Les vœux que les conseils des facultés de lettres font publier dans la RIE de 1904 à 1906 témoignent d'une grande unanimité des réactions. Ils demandent la suppression de toutes les bourses affectées à l'Université de Paris, autres que les pensions des normaliens, et leur redistribution aux universités de province de telle sorte que le nombre de boursiers de licence affectés en province soit au moins le même que celui des normaliens recrutés. La critique est très vive : Paris "vole " les étudiants, les " aspire ", et ce d'autant plus que la division des tâches entre l'université et l'École leur offre une double formation, scientifique et pédagogique, que les facultés de province ne peuvent mettre en place. La réforme de 1903 qui, pour ses contempteurs, améliore la situation de la seule université de Paris, déjà privilégiée, semble donc défaire ce qu'avait permis la loi de finances du 29 décembre 1876, la création des bourses de licence en nombre suffisant pour attirer les étudiants vers les facultés des lettres de province, puis des bourses d'agrégation qui devaient, elles aussi, favoriser la décentralisation de la préparation du concours.

L'autre enjeu de la réforme de l'ENS est l'affectation des emplois de maîtres de conférences supprimés par la fusion de l'école et de l'université, puisque l'article 10 du décret de 1903 stipulait que leurs titulaires relevant du cadre de l'ENS entraient dans les cadres de l'université de Paris. Considérant que la réunion renforce les positions de l'université parisienne et de ses professeurs, les doyens des facultés de lettres de province demandent que ces postes leur soient transférés, pour combler l'écart dû au fait que Paris concentre déjà les chaires, les anciennes et celles qui, depuis la réforme, ont été créées par l'État ${ }^{17}$. Les universités de province ont, elles, en effet, plutôt obtenu de l'État des postes de maîtres de conférence ou de chargés de cours, et en nombre moindre que Paris. En revanche, proportionnellement au nombre d'étudiants, elles en ont financé sur leurs subsides plus que la capitale. Par ailleurs, le fait que les dotations de l'État n'aient pas augmenté en proportion de la réduction des dépenses qui, selon les dispositions de la loi, sont imputées aux facultés, et que, en outre, elles soient émiettées entre les établissements pose la question du coût financier de la décentralisation et de sa prise en charge. Aussi, dans les vœux qu'elles déposent, les facultés des lettres qui font état de leurs "besoins si considérables " et de leurs "ressources si aléatoires " se sentent-elles habilitées à demander à leur profit le transfert des crédits de l'École devenus disponibles pour soutenir la concurrence de Paris. Cette cause obtient le large soutien de l'Association du personnel enseignant des facultés des lettres créée en 1907, à l'instar de celles de sciences, pour obtenir un tableau unique de titularisation et d'avance-

17. Voir Annexe 2, "Les facultés des lettres des universités françaises ", RIE, 1910, 1, p. 107-108. 
ment des professeurs et maîtres de conférences et poser la question de l'inégalité des rémunérations entre Paris et la province.

On voit donc que les privilèges de Paris sont dénoncés à plusieurs niveaux. La cible est la Sorbonne, coupable de concentrer étudiants et maîtres ${ }^{18}$ et de considérer les crédits de l'État comme les siens propres. Mais la critique n'épargne pas totalement le ministère et la direction de l'enseignement supérieur, accusés d'être " complices " de la Sorbonne, de la laisser " décréter tout à son profit " et renforcer son " monopole ". Au demeurant, cette opposition ne créait pas vraiment de surprise. Le ministre Chaumié avait en vain tenté de la prévenir en assurant dans les derniers mots du rapport précédant la présentation du décret de la réorganisation de l'ENS que « les mesures projetées ne [lèseraient] pas les intérêts des universités de province ". Les chiffres publiés dans la RIE pour les années 1905/06, 1906/07, 1907/1908 renvoient une tout autre réalité : le nombre de boursiers de licence en province est inférieur à celui qu'il était avant la réforme et malgré l'éparpillement entre les facultés, certaines d'entre elles n'en ont aucun ${ }^{19}$.

Le conflit à propos de l'ENS révèle donc les illusions de la décentralisation intellectuelle au nom de laquelle Louis Liard justifiait la loi de 1896. Si l'autonomie doit être le nouveau régime des universités, la demande des facultés de lettres de province est que ce soit une vraie autonomie. Car si la loi les rend en partie maîtresses de leurs enseignements, elles ne le sont ni de la gestion du personnel ni des recrutements, l'administration supérieure gérant les carrières des professeurs titulaires et maîtres de conférences et les recteurs nommant aux emplois de maîtres de conférences et chargés de cours, quels que soient les fonds sur lesquels ils étaient rétribués. Elles dénoncent donc une autonomie qui revient à livrer les facultés à elles-mêmes ou au "régime des coteries ${ }^{20}$ ". Dans les deux cas, c'est l'échec du projet républicain de réaliser, au service de la connaissance, l'unité du tout, l'université, et des parties, les facultés, celles-ci se disputant pour équilibrer leur propre budget les faveurs de tous ceux qui peuvent les financer : municipalités, conseils généraux et Chambres de commerce, associations et particuliers. Si, dans les faits, il s'avère que s'est souvent resserrée la collaboration avec les villes et les conseils généraux, les tableaux des donations et legs aux universités provinciales publiés par la RIE montrent que les financements se distribuaient très inégalement selon le type de facultés considéré ${ }^{21}$. Ils étaient aussi aléatoires, bien qu'ils fussent nécessaires, surtout pour les petites facultés, Aix, Clermont, Caen, Poitiers... On le voit à la mobilisation par

18. Bouglé, Célestin, "La réforme des agrégations et la réorganisation de l'ENS ", RIE, 1904, 1, p. 45-49. Les professeurs parisiens sont même suspectés, pour garder leur chaire, d'entretenir la pléthore des candidats à l'agrégation dans la capitale.

19. Voir Annexe 3.

20. SimYAN, "Rapport du budget de l'Instruction publique ", RIE, 1903, 2, p. 520-528.

21. "L'enseignement supérieur dans les départements : donations et legs des universités provinciales ", RIE, 1905, 2, p. 98-117. 
exemple que provoque en 1911 la décision du conseil général des Bouches du Rhône de supprimer le cours de lettres organisé à Marseille, repris finalement par la Société des Amis des lettres.

Alors que la réunion de l'ENS et de l'université de Paris soulève indéniablement des problèmes, l'émoi qu'elle provoque dans les rangs provinciaux montre combien l'image, déjà ancienne, des malheureuses facultés structure la lecture de la réalité. La métaphore de l'abandon en est caractéristique. Dans une période où la loi sur les enfants moralement abandonnés est récente, l'argument de l'abandon moral dont l'État est rendu responsable fait mouche. Mais l'insistance sur le manque de ressources $^{22}$, le manque de bibliothèques et d'ouvrages à la disposition des professeurs et des étudiants, qui s'affirment pourtant bien moins exigeants que leurs collègues de sciences, la description des locaux en mauvais état, des pièces basses et moroses ${ }^{23}$, s'inscrivent dans le même registre de la déploration, entretenant le sentiment que les facultés de lettres sont vraiment mal loties. Cependant, et de façon apparemment paradoxale, le registre de la doléance alterne avec celui de l'autocélébration. Chaque faculté valorise ses effectifs et ses activités et à travers les rapports annuels que les doyens des facultés de lettres remettent au conseil de l'Université ${ }^{24}$, une autre image vient en surimpression de la première : malheureuses certes, mais hargneuses et prêtes à avoir leur part du gâteau. Telle est en fait la double image que les personnels des facultés de lettres de province construisent par leurs discours et écrits repris par la presse, mais aussi dans l'élection au comité consultatif du CSIP (Conseil supérieur de l'Instruction publique) de Clédat, que les papiers à la fois alarmistes et indignés qu'il avait livrés à la RIE ont désigné comme le représentant le plus qualifié des intérêts mêlés de la province et de l'enseignement des lettres. Car la définition même des missions de l'enseignement universitaire des lettres est, dans les faits, suspendue à l'avenir des facultés de province.

\section{Les facultés de province et la recomposition de l'enseignement supérieur des lettres}

" On ne peut concevoir l'existence d'universités provinciales si on ne les suppose pas animées par l'idée et la passion de la science. La science est

22. Dans sa séance de rentrée en 1904 évoquée déjà ci-dessus, le conseil d'université de Dijon fait par exemple état d'une situation critique qu'il impute à l'État, mais qui risque d'être particulièrement préjudiciable à la faculté de lettres, puisque, dans des conditions budgétaires déjà difficiles, les crédits internes et les aides extérieures sont en priorité dirigés vers les facultés dont l'activité - recherche comprise - est soutenue.

23. Desdevises Du DEzERT " Chronique de l'enseignement ", RIE, 1899, 1, p. 63

24. Les rapports annuels des conseils valorisent, face aux autres doyens de l'université, les marques de "rayonnement ": inauguration, en présence de délégués d'universités étrangères, de locaux, conférences, déplacements, publications, fondation des instituts français de Florence, de Madrid, de Saint- Petersbourg ou de l'école française de Constantinople. 
même la seule force qui puisse les créer et les maintenir ${ }^{25}$ ", avait déclaré Dumont. De cette logique qui, de 1877 à 1896, a été sous-jacente à la réforme universitaire, la réforme de l'ENS, selon le rapport qu'en présente le ministre Chaumié, est en quelque sorte l'aboutissement, puisqu'en confiant la préparation pédagogique des professeurs à l'École, elle a pour objectif de permettre aux facultés des lettres de se dégager de cette tâche et de se consacrer à l'initiation scientifique des étudiants et à la diffusion des résultats de la science, afin de devenir ces puissants foyers d'étude et de recherche que les réformateurs avaient l'ambition de constituer dans l'intérêt de la nation. Or, comme dans les facultés de lettres, une grande part de cette activité scientifique s'organisait autour de l'agrégation, l'enjeu était pour les facultés de province d'en assurer la préparation. En effet, pilier de toutes les études universitaires, la préparation du concours était aussi souvent le vivier des chercheurs, et ce d'autant plus que, depuis 1904, le DES (diplôme d'études supérieures), initiant les candidats au professorat aux méthodes de la recherche, avait été instauré dans toutes les disciplines comme étape préparatoire. Enfin, les cours d'agrégation, par leur ancienneté et leur dimension symbolique, faisaient le prestige des facultés de lettres.

Cela supposait, pour les facultés de province, qu'elles puissent attirer une " clientèle sérieuse ", celle des rhétoriques supérieures ${ }^{26}$ de leur ressort dont il est alors envisagé de transférer aux facultés la préparation au concours des bourses, mais surtout la clientèle des boursiers, ce qui explique qu'ils aient été l'enjeu de la réforme de l'ENS. S'attirer les boursiers, et l'expression est d'autant plus juste que les boursiers reçus au concours choisissent la faculté où ils s'inscrivent, c'est en effet, comme le formule nettement Clédat, avoir sa part de " bons " élèves ${ }^{27}$. Mais le concours unique mis en place à partir de 1905 revient dans les faits à affecter à la province les étudiants classés après les normaliens, les candidats classés les premiers, c'est-à-dire, ceux considérés comme les "meilleurs élèves " choisissant, sauf exception, Paris et l'École normale. Partant de la probabilité - qu'ultérieurement les faits n'ont pas systématiquement vérifiée - que les normaliens réussissent l'agrégation dans une proportion plus élevée que les candidats de province ${ }^{28}$, les professeurs de province craignent qu'à terme leurs préparations de l'agrégation,

25. Dumont, Albert, « Note sur l'enseignement supérieur en France », RIE, 1884, 2, p. 193234.

26. Appellation ancienne des Premières supérieures pour les classes sui préparaient au concours de l'ENS.

27. CLEDAT, Léon, "Enquête sur la spécialisation des facultés de lettres ", RIE, 1909, 1, p. $152-153$.

28. MonOD, Gabriel, « La réforme de l'ENS ", Revue historique, 1904, T. 84, p. 308-313. Selon les chiffres donnés par le ministre Chaumié lui-même et reproduits dans cet article, pour les dix dernières années, Paris avait eu, sur 284 boursiers, 94 agrégés reçus en lettres, tandis que sur les 457 boursiers de province, 64 avaient été reçus. Si les probabilités de réussite des bousiers parisiens étaient supérieures, le ministre, quant à lui, tablait sur le fait que le nombre d'étudiants en lettres se préparant à l'agrégation en province augmenterait parce que l'université de Paris ne présenterait pas d'autres candidats à l'agrégation que les élèves de l'EN. 
dévaluées, soient désertées, et que la fonction d'ateliers scientifiques qu'elles conféraient aux facultés s'en trouve menacée. En l'absence d'une répartition équitable des candidats à l'agrégation, la formation universitaire des professeurs de l'enseignement secondaire dans les disciplines littéraires semble s'acheminer, pour eux, vers la situation suivante : à la faculté de Paris le monopole de la préparation des agrégés; aux facultés de province, la préparation des licenciés, comme le note avec ironie A. Balz dans la Revue universitaire $^{29}$. Certains d'ailleurs ne se privaient pas de penser : et aux moins favorisées de toutes, les activités " compensatoires " de l'extension universitaire, que chaque faculté organisait sur ses propres forces ${ }^{30}$, et en conséquence moins sûres que celles qui, débouchant sur la délivrance de grades nationaux, étaient financées par l'État. On voit ainsi comment affleurent dans ces débats les rivalités induites par la hiérarchie interne des facultés provinciales restée vingt ans après la réforme de 1896 globalement inchangée. La crainte est que la distinction des activités ne l'accroisse et elle attise les rivalités. Les " appétits " de Lyon inquiètent par exemple Grenoble et Nancy ${ }^{31}$.

L'inéluctabilité du déclin des facultés de lettres de province est brandie comme si elle était implicitement contenue dans leur passé de " malheureuses " facultés. Les scénarios que présentent à titre individuel les auteurs qui, de leur province, écrivent à la $R I E$ sont tous pessimistes. Leur enlever les préparations à l'agrégation, c'est les affaiblir inévitablement; mais en même temps les maintenir est prendre le risque que la fonction professionnelle prenne le pas sur la fonction scientifique. Le débat ${ }^{32}$ réactive donc les questions que l'enquête parlementaire de 1899 avait posées, celles de la professionnalisation des facultés de lettres que la réforme de l'agrégation rend particulièrement sensible et de la spécialisation des études, c'est-à-dire la concentration géographique des préparations pour créer des pôles en mesure d'empêcher que les agrégations ne deviennent " des concours entre établissements parisiens ${ }^{33}$ ". Pour les " petites facultés ", la spécialisation réalisée par le regroupement de la préparation des agrégations, voire des licences, comme le propose Lapie pour la philosophie, fait

29. BALZ, André, "Chronique du mois ", $R U, 1904,1$, p. 58.

30. CompaYRÉ, Gaston, "Séance de rentrée des universités ", RIE, 1909, 1, p. 49-53. Devant l'auditoire de l'université de Lyon, Compayré déclare : «L'extension universitaire convient surtout aux universités de moindre importance qui ont un surcroît de forces à dépenser parce que leurs occupations normales ne les absorbent pas toutes et qui, par suite, sont disposées à rechercher les besognes supplémentaires, les occupations d'à côté. "

31. MARTIN, Arthur, "Réponse à l'enquête sur la spécialisation des facultés ", RIE, 1909, 1, p. 77-82. Le doyen de Nancy exprime le regret que « la faculté de Lyon soit grandement favorisée ".

32. "Enquête sur la spécialisation des facultés de lettres ", RIE, 1908, 2, p. 340-346. La revue ouvre l'enquête après la publication d'une lettre de Clédat favorable à la spécialisation. Cependant, la question avait déjà été soulevée en 1903 par le groupe parisien de la Société pour l'étude des questions d'enseignement supérieur, inquiète des conséquences éventuelles de la loi militaire sur les effectifs étudiants. Ce passage de l'article est construit à partir des réponses de toutes les facultés provinciales que les numéros de la RIE publient tout au long du deuxième semestre de 1908 et de l'année 1909.

33. BalZ, André, "Chronique du mois ", $R U, 1904,1$, p. 437. 
planer le risque que soient supprimés des enseignements et redistribués les moyens qu'ils mobilisent au profit des cinq ou six centres déjà les plus dotés. En même temps, dans certaines disciplines enseignées depuis peu en lycée, comme l'espagnol et l'italien, le nombre d'élèves est encore si faible que la préparation à l'agrégation se concentre déjà dans les facultés des académies où ces langues sont parlées, sans qu'elles échappent pour autant au risque d'être dans l'impossibilité, faute d'étudiants, d'organiser une préparation " complète ". Le fait que l'État, en 1908, déclare se réserver la possibilité de ne pas ouvrir le concours de recrutement au professorat d'arabe, d'espagnol et d'italien, est éloquent et provoque une vive émotion à la faculté des lettres de Grenoble qui a un important contingent d'italianisants, de même qu'à Toulouse et Montpellier qui se disputaient l'enseignement supérieur de l'espagnol.

Certes, les professeurs parisiens s'inquiètent aussi du poids que prennent dans l'enseignement supérieur les tâches liées aux examens et à la " professoralisation ", mais ceux de province s'y trouvent encore plus assujettis, dans la mesure où ils sont en trop petit nombre pour préparer l'agrégation. Qui plus est, déplorent-ils, alors que la préparation de l'agrégation monopolise leurs forces et réduit leur rôle à celui de préparateur ${ }^{34}$, elle ne suffit pas à retenir les candidats en province. Ainsi les professeurs de province se trouvent-ils directement confrontés à la difficulté de tenir l'équilibre entre la préparation au professorat, la diffusion de la haute culture et la production scientifique. Dans ces conditions, le décret de 1903 qui consacre - sur le papier - la division du travail entre « l'Université, atelier scientifique et l'École normale séminaire pédagogique ${ }^{35}$ " est perçu comme un avantage de plus accordé aux " sorbonnards " enviés pour être libres de choisir, en fonction de leurs talents propres et de leurs centres d'intérêt, l'enseignement ou/et la recherche.

Le début du $\mathrm{Xx}^{\mathrm{e}}$ siècle est donc ce moment où la renaissance universitaire de l'enseignement des lettres se construit à travers les tensions entre enseignement et recherche, culture générale et spécialité, voire entre disciplines, tensions qui se trouvent exacerbées dans les facultés de province ramenées toujours à la nécessité d'obtenir les moyens - humains et matériels - nécessaires pour fixer professeurs et étudiants - et le terme de fixer dit bien qu'il s'agit d'éviter que les uns et les autres ne partent à Paris. Avec les moyens dont elles disposent, parmi lesquels ceux alloués par l'État restent maigres, elles se plaignent de devoir jouer en même temps la carte de

34. BouRDON, Benjamin, "Enquête sur la spécialisation des facultés de lettres ", RIE, 1909, 1, p. 363-367. Gérard-Varet dénonce "l'obsession de l'agrégation ", 1909, 2, p. 148. Loth emploie, quant à lui, l'expression de "fours à agrégation ", 1908, 2, p. 530. Cependant, ces remarques ne sont pas nouvelles; A. Lebègue écrivait en 1884 à propos de l'agrégation : " Nous n'avons plus le temps de préparer d'autres cours ", RIE, 1884, 1, p. 535-542.

35. Bouglé, Célestin, "La réforme des agrégations et la réorganisation de l'ENS ", RIE, 1904,1, p. $45-63$. 
la préparation à l'agrégation, celle de l'orientation de plus en plus spécialisée - scientifique - des études où sont promues de nouvelles disciplines et instituées de nouvelles licences, et celle du déploiement le plus large possible, c'est-à-dire de l'extension universitaire et des enseignements généraux. Mais ce qui apparaît peu dans les bilans présentés par leurs doyens, est que, même si elles ont eu une crise à surmonter telle que leur développement n'a pas été aussi rapide qu'elles l'eussent souhaité, à moyen terme, elles ont tiré profit de cette recomposition. Le développement des enseignements existants, l'introduction de nouveaux savoirs, la création de diplômes et de postes les ont vivifiées, leur fonction de recherche s'est affirmée dans des créneaux qu'elles occupaient souvent seules et avec elles, les positions scientifiques et institutionnelles de leurs professeurs. Ainsi, se portaient-elles mieux en 1913 qu'en 1896. Leur renaissance se profilait, comme l'ont confirmé les travaux historiques consacrés à l'enseignement supérieur français ${ }^{36}$. Finalement, le principe d'émulation qui était pour les réformateurs le contrepoint de l'inégalité des universités et dont ils attendaient qu'il " [tourne] au bien des études, de la science et du pays ${ }^{37}$ " a joué aussi en leur faveur, même s'il n'a pas complètement échappé au risque de mettre en concurrence des intérêts particuliers. Chaque faculté, on l'a noté, s'est organisée, mettant au point, en fonction de ses atouts spécifiques, ses propres stratégies offensives pour obtenir des crédits, avoir des cours à assurer. Clermont peut, dès lors, parler de " réveil ${ }^{38}$ " et Grenoble, fêtant son centenaire en 1910, reprend triomphalement le mot de Dumont en 1879 « Il n'y a pas de petites facultés ${ }^{39}$ ". Au vu de l'annexe 1 présentée ci-dessous, la formule est un peu outrée.

Dans la foulée de la loi de 1896, entonner le refrain des malheureuses facultés, dans un contexte où les réformateurs envisageaient la constitution de pôles régionaux pour faire vivre la décentralisation intellectuelle, a été pour les universitaires de province une arme de négociation face à l'establishment parisien. Parallèlement, exploitant les moyens que leur avait donnés la loi de 1896, les facultés des lettres prenaient en mains leur croissance ou leur rétablissement, contribuant, comme le montre le cas de Rennes, à la prospérité de l'enseignement supérieur des lettres à côté des études prestigieuses de droit et de médecine. Mais le dossier de l'autonomie était loin d'être refermé et aujourd'hui, alors qu'en application de la loi relative aux libertés et responsabilités des universités, de nouveaux statuts sont entrés en vigueur après avoir suscité des analyses contradictoires, on est frappé par la perspicacité avec laquelle les contemporains de la création des universités de province en avaient cerné les enjeux majeurs.

36. Particulièrement : BuRneY, John M., Facultés et étudiants dans la France provinciale du $19^{e}$ siècle, toulouse, Presses universitaires du Mirail, 1988.

37. LIARD, Louis, "Les Universités françaises ", Statistique de l'enseignement supérieur, p. I-XXXXX, Paris, Imprimerie nationale, 1899.

38. Audollent, Alphonse, "Le réveil des universités françaises " RIE, 1918, p. 443-452.

39. Morillot, Paul, "Centenaire de la Faculté des lettres de Grenoble; une faculté des lettres d'aujourd'hui ", RIE, 1910, 2, p. 443-457. 
Annexe 1 - Effectifs des étudiants immatriculés en lettres

(Sources : Statistique de l'enseignement supérieur, Faculté des Lettres, 1898, p. 317; “ Les Universités françaises en 1912-1913 », RIE, 1914, 2, p. 47 - 213. NB : Caen et Toulouse ne précisent pas s'il s'agit d'étudiants immatriculés ou inscrits)

\begin{tabular}{|l|l|l|}
\hline & $1897-1898$ & $1912-1913$ \\
\hline Paris : & 1697 & 3107 \\
\hline Aix-Marseille & 44 & 33 (étrangers non compris) \\
\hline Besançon & 67 & 125 \\
\hline Bordeaux & 139 & 271 \\
\hline Caen & 122 & 172 \\
\hline Clermont & 90 & 95 (dont 29 élèves bénévoles) \\
\hline Dijon & $\begin{array}{l}52(+102 \text { pour les lectures } \\
\text { allemandes et anglaises) }\end{array}$ & 183 \\
\hline Grenoble & 77 & 636 (étrangers inclus) \\
\hline Lille & 285 & 353 \\
\hline Lyon & 200 & 379 (étrangers non compris) \\
\hline Montpellier & 90 & $?(119$ étudiants étrangers) \\
\hline Nancy & 112 & 207 (dont 69 étudiants étrangers) \\
\hline Poitiers & 77 & 206 (233 au total) \\
\hline Rennes & 102 & 141 (inscrits) \\
\hline Toulouse & 112 & 379 \\
\hline Ecole d'Alger & 44 & 198 \\
\hline
\end{tabular}


Annexe 2 - Liste des chaires et enseignements créés par l'État et les universités, 1904-1910

(Source: "Les facultés des lettres des Universités françaises", RIE, 1910, 1, p. 107-108)

\begin{tabular}{|l|l|l|l|}
\hline Chaires créées & \multicolumn{2}{|l|}{ par l'État } & L'Université \\
\hline & Paris & 11 & \\
\hline & Lille & 2 & 1 \\
\hline & Lyon & & 2 \\
\hline & Montpellier & 1 & \\
\hline & Rennes & 1 & \\
\hline Cours et conférences & Paris & 13 & 3 \\
\hline & Aix & & 2 \\
\hline & Bordeaux & 1 & 2 \\
\hline & Caen & 1 & 1 \\
\hline & Clermont & 1 & 1 \\
\hline & Grenoble & 1 & 3 \\
\hline & Lille & 1 & \\
\hline & Lyon & & 4 \\
\hline & Montpellier & 2 & 2 \\
\hline & Nancy & & \\
\hline & Poitiers & & 3 \\
\hline & Rennes & & 2 \\
\hline & Toulouse & 2 & \\
\hline & & & 1 \\
\hline
\end{tabular}


Annexe 3 - Répartition des boursiers entre les facultés de lettres de province et Paris (Source : Léon Clédat, "Statistique des boursiers de licence ", RIE, 1908, 1, p. 304)

\begin{tabular}{|c|c|c|c|}
\hline Facultés & $1907 / 08$ & $06 / 07$ & $05 / 06$ \\
\hline Paris & 35 & 35 & $32^{*}$ \\
\hline Total province & 18 & 23 & 20 \\
\hline dont & & & \\
\hline Aix & 0 & & \\
\hline Besançon & 0 & & 3 \\
\hline Bordeaux & 1 & 6 & \\
\hline Clermont & 0 & & 3 \\
\hline Dijon & 0 & & \\
\hline Lille & 3 & 1 & \\
\hline Lyon & 8 & 9 & 1 \\
\hline Poitiers & 1 & & \\
\hline Rennes & 1 & & \\
\hline Toulouse & 1 & & \\
\hline
\end{tabular}

*Plus 1 non normalien en 1905-1906 (Pour mémoire, avant 1903, la moyenne des boursiers de province était de 30 ; l'ENS en avait 20 et la faculté des lettres de Paris 12 . Comme on le constate, les informations de Clédat sont incomplètes. C'est pourquoi la somme de chaque colonne est inférieure au total qu'il donne pour les boursiers de province. 


\section{RÉSUMÉ}

"Les malheureuses facultés de province ", par cette expression empruntée à Renan, un membre de la Société pour l'étude des questions de l'enseignement supérieur s'inquiète, dans la Revue internationale de l'Enseignement, de la situation des facultés de lettres depuis la réforme de 1896 . Celles-ci considèrent en effet qu'elles n'ont pas les moyens de soutenir la concurrence de l'Université parisienne : elles manquent d'étudiants et donc de ressources financières. La réforme de l'École Normale Supérieure en 1903 accentue, selon elles, ces inégalités. Dénonçant cette situation, le personnel des facultés de lettres de province recherche avec détermination des solutions : mesures administratives pour accroître le nombre d'inscrits, sélection des diplômes préparés, spécialisation par pôles... Dix ans plus tard, le réveil semble s'amorcer.

\section{ABSTRACT}

A member of the Society for the study of questions concerning higher education wrote in the Revue international de l'Enseignement about the "unfortunate provincial faculties", an expression borrowed from Ernest Renan. He was concerned about the situations of the Arts Faculties since the reform of 1896. These faculties considered that they didn't have the means to compete with the University of Paris: they lacked students and therefore financial resources. The reform of the École Normale Supérieure in 1903 increased, in their opinion, these inequalities. The employees of the provincial Arts Faculties looked, with determination, for solutions while denouncing the situation. These solutions concerned administrative actions to increase enrolment, selection of degrees offered, specialisation by centres of development, etc. Ten years after changes started to happen. 